\title{
RESULTS OF DOUBLE STARS OBSERVATIONS AT NIKOLAEV OBSERVATORY
}

\author{
D.V.Bodryagin, N.V.Maigurova \\ Research Institution "Mykolaiv Astronomical Observatory", \\ Observatorna St.1, 54030, Mykolaiv, Ukraine
}

\begin{abstract}
The results of double stars observations at Nikolayev Observatory during 2013-2014 are presented. The Washington Double Stars Catalogue (WDS) was used for observation program composing. The observation volume was obtained with usage two telescopes equipped CCD-cameras. Precise modern epoch CCD-observations allowed to make new proper motion values. The position angles and separations for 214 double stars were measured. Results are going to be submitted to WDS.
\end{abstract}

Keywords: Stars: double stars, binary stars, proper motions

\section{Introduction}

The main reason to be interested in double stars is a possibility to obtain total mass of a double-star system from studying their relative motions. Mass estimates are of huge importance to theorists on stellar evolution. According to modern double stars researches, the percentage of stars included into double or multiple systems is in range from 30 to $70 \%$. The main source of information about these objects is the Washington Double Star Catalog (WDS) [1]. WDS contains more than 132,000 objects on July, 2015 each of which gives measures for the positional angle and separation of two components. Visual double stars are two that appear close together in the sky visually, but are not necessarily anywhere near one another in space. If visual doubles are gravitationally attached they are called visual binaries. Similar proper motions for both components is one of criteria for distinguish them. If the difference of their proper motions is small comparing to their common proper motion, the pair is probably physical. The proper motions for $1.5 \%$ of main component and for $20 \%$ second component are absent in WDS catalog. The most of the doubles are available for observations with small telescopes. That's why we have performed double stars observations in Nikolaev.

\section{Observations: Instrumentation, Program}

The observations were made at two telescopes which were created in the RI NAO: mobile multi-channel automatic telescope (Mobitel) with $\mathrm{D}=500 \mathrm{~mm}, \mathrm{~F}=3000 \mathrm{~mm}$ and Axial meridian circle (AMC) with $\mathrm{D}=180 \mathrm{~mm}$, $\mathrm{F}=2500 \mathrm{~mm}$.

Both telescopes are equipped with the CCD cameras and getting images in drift scan mode. We have obtained frames with scale $1.32^{\prime \prime}$ and $0.83^{\prime \prime}$ per pixel and field of view $24^{\prime} \times 28^{\prime}$ and $42.6^{\prime} \times 42.6^{\prime}$ for AMC and Mobitel, respectively. These parameters allow us to have enough reference stars for astrometric reductions and obtaining positions of the stars with high accuracy.

For observational program we selected WDS objects which are most apropriate to be observed by our telescope and in the expected time frame (the full time of exposure is $102^{\mathrm{s}}$ and $85^{\mathrm{s}}$ at equator). For the main selection criteria we used a magnitude limit of $17^{\mathrm{m}}$ and $14.5^{\mathrm{m}}$ and a separation bigger than two FWHM.

The regular observations of double stars were carried out during 2013-2014. The statistical information about volume of observations is given in table 1 . There are not only program objects in column 4; we also selected other WDS doubles from the other observations, which appeared in the imaged field.

Table1. Statistics of observations

\begin{tabular}{|l|l|l|l|}
\hline Period & Telescope & $\Sigma$ stars & Mean N $^{*}$ \\
\hline 2013 & AMC & 2420 & 4 \\
\hline 2014 & AMC & 1848 & 3.5 \\
\hline 2013 & Mobitel & 124859 & 8 \\
\hline 2014 & Mobitel & 87767 & 9 \\
\hline
\end{tabular}

*The average number of star observations

\section{Processing and Results}

Processing of obtained observations included two steps: getting astrometrical parameters (coordinates, proper motions, magnitudes) and measures of double stars parameters (position angle and separation).

Astrometric reductions for equatorial coordinates of objects were performed with Astrometrica software [2]. Connection model between the tangential and the measured coordinates was polynomial 2 nd order for AMC and polynomial 4th order for Mobitel. The catalogs UCAC2 [3] and UCAC4 [4] were used as reference catalogs. After processing whole volume of observations the coordinates in right ascension and declination at mean observational epoch were averaged. The mean standard errors of catalog position were about 30 mas in both coordinates and that allows us to obtain new proper motions with accuracy about 4-5 mas/year when the catalog USNO A2.0 was used as source of second epoch positions. Then we cross-matched our positional data with WDS catalog and other astronomical catalogs for calculations new proper motions of the primary and secondary double components and for selection all WDS doubles which were appeared in the imaged field. The results of search WDS double stars and following cross-matching are summarized in table 2. Cross-matching was made with usage 
service CDS-Upload X-Match realized by TOPCAT software [5].

Table 2. Results of cross-matching

\begin{tabular}{|l|l|l|l|l|l|l|}
\hline & WDS & $\begin{array}{c}\text { USNO } \\
\text { A2.0 }\end{array}$ & PPMXL & 2mass & UCAC4 & TYCHO2 \\
\hline AMC & 127 & 118 & 123 & 127 & 127 & 74 \\
\hline Mobitel & 234 & 216 & 222 & 234 & 230 & 55 \\
\hline
\end{tabular}

The comparison of obtained proper motions has shown a good agreement with other sources. The mean external accuracy of the obtained proper motions is about 8 mas/year in both coordinates based on the comparison with other catalogs (Tycho2, PPMXL, UCAC4, WDS).

The comparison results of obtained proper motions with WDS proper motions are shown in figure 1.
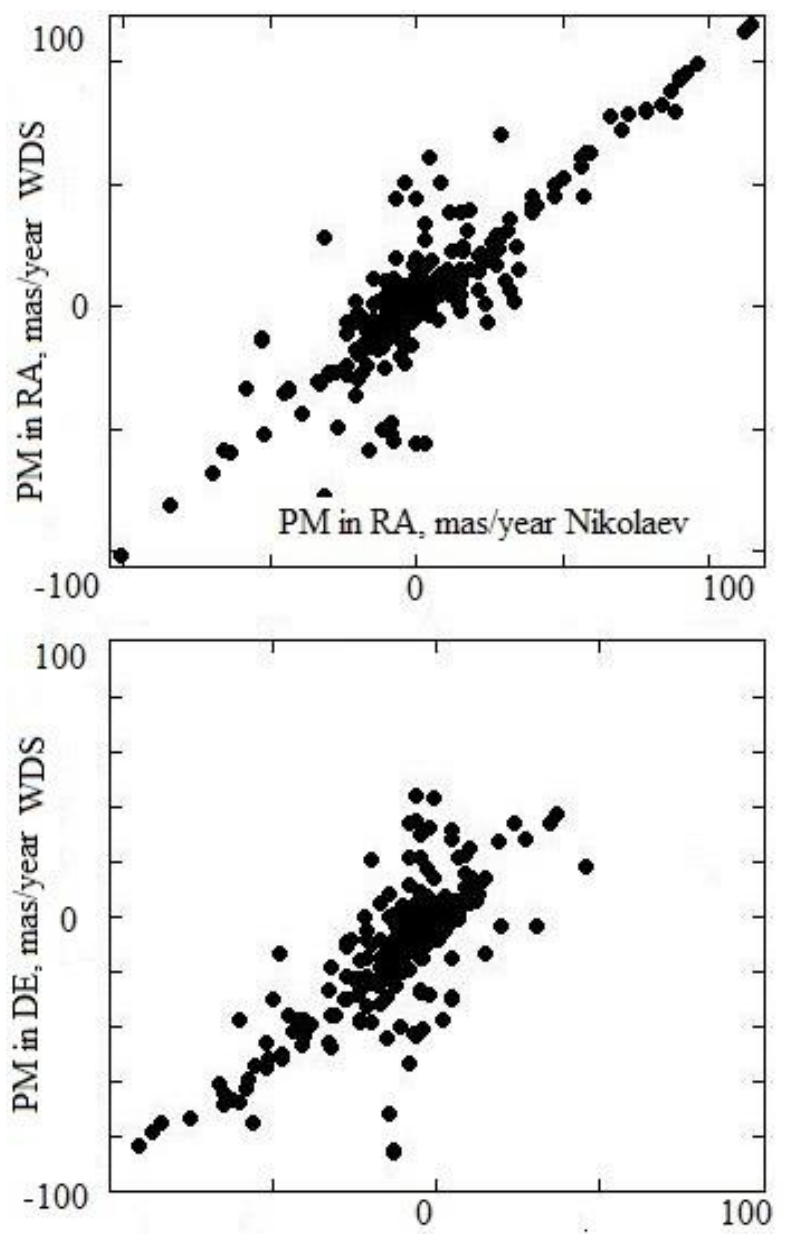

$\mathrm{PM}$ in DE, mas/year Nikolaev

Figure 1: Obtained proper motion vs WDS proper motions (top - right ascension, bottom - declination).

The figure 1 have shown that criteria of common proper motions for distinguishing visual binary stars work better for stars with high proper motions, when values of their error much less than values of proper motions.

Measures of double stars were made with usage REDUC software [6]. We used previously determined exact values of orientation angle and image inclination regarding the celestial equator from astrometric reductions for calibration. Unfortunately, the AMC limiting magnitude fallen down significantly by the technical reasons and we can't see the secondary component in most frame there. We have measured only observations obtained at Mobitel.

There were obtained 1802 positions for 199 WDS stars. The mean number of measurements of a star is about 8 . The distributions of observations in stellar magnitudes and magnitude differences between main and secondary component measured by REDUC are given in figure 2.
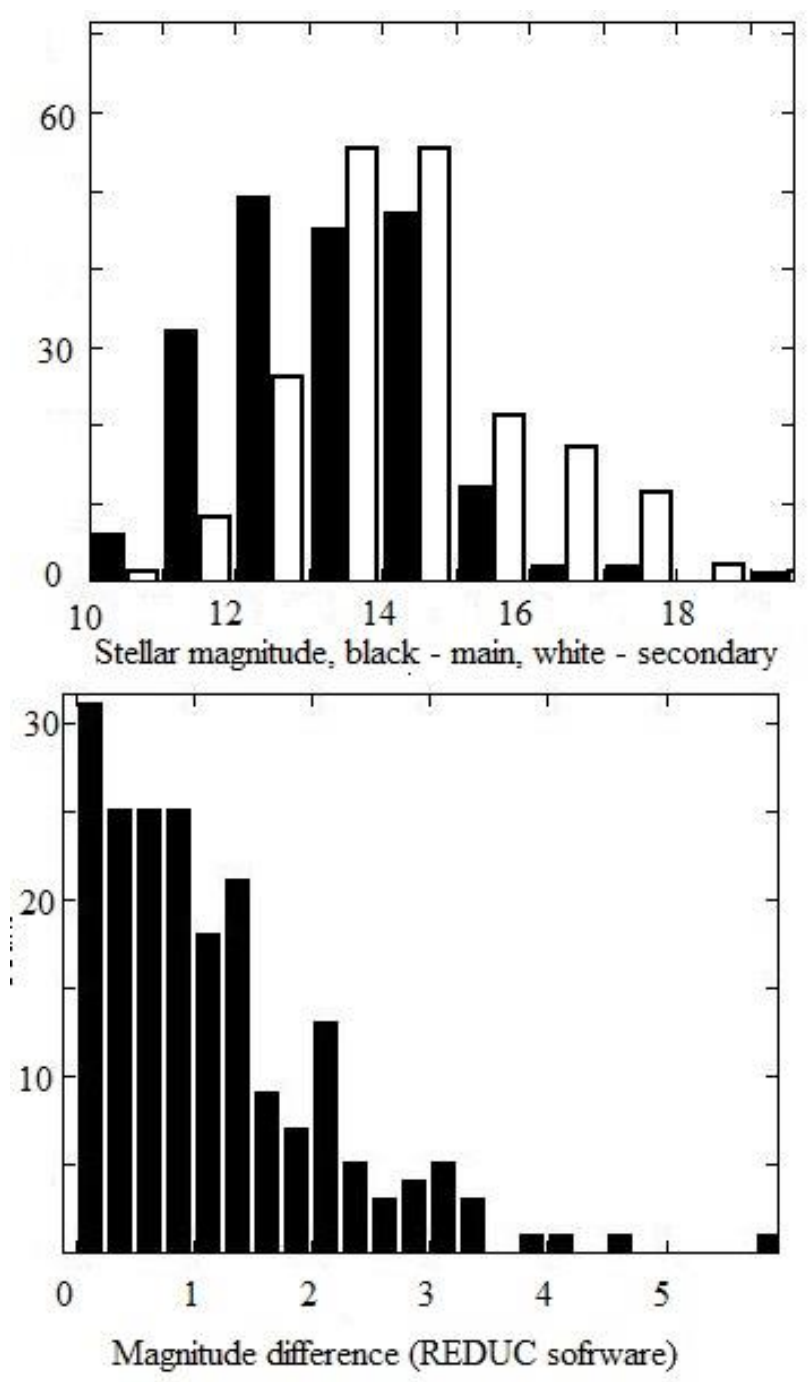

Figure 2: Distribution of double stars in stellar magnitude (top - stellar magnitude of main (black column) and secondary (white column), bottom - magnitude difference between the components, obtained REDUC software)

The position angle (PA) of the secondary with respect to the primary and angular distance between the two stars (separation) between components were measured. The mean standard error of PA is $0.5^{\circ}$, separation - $0.15^{\prime \prime}$. The distribution of standard errors in PA and separation are shown in figure 3 . 

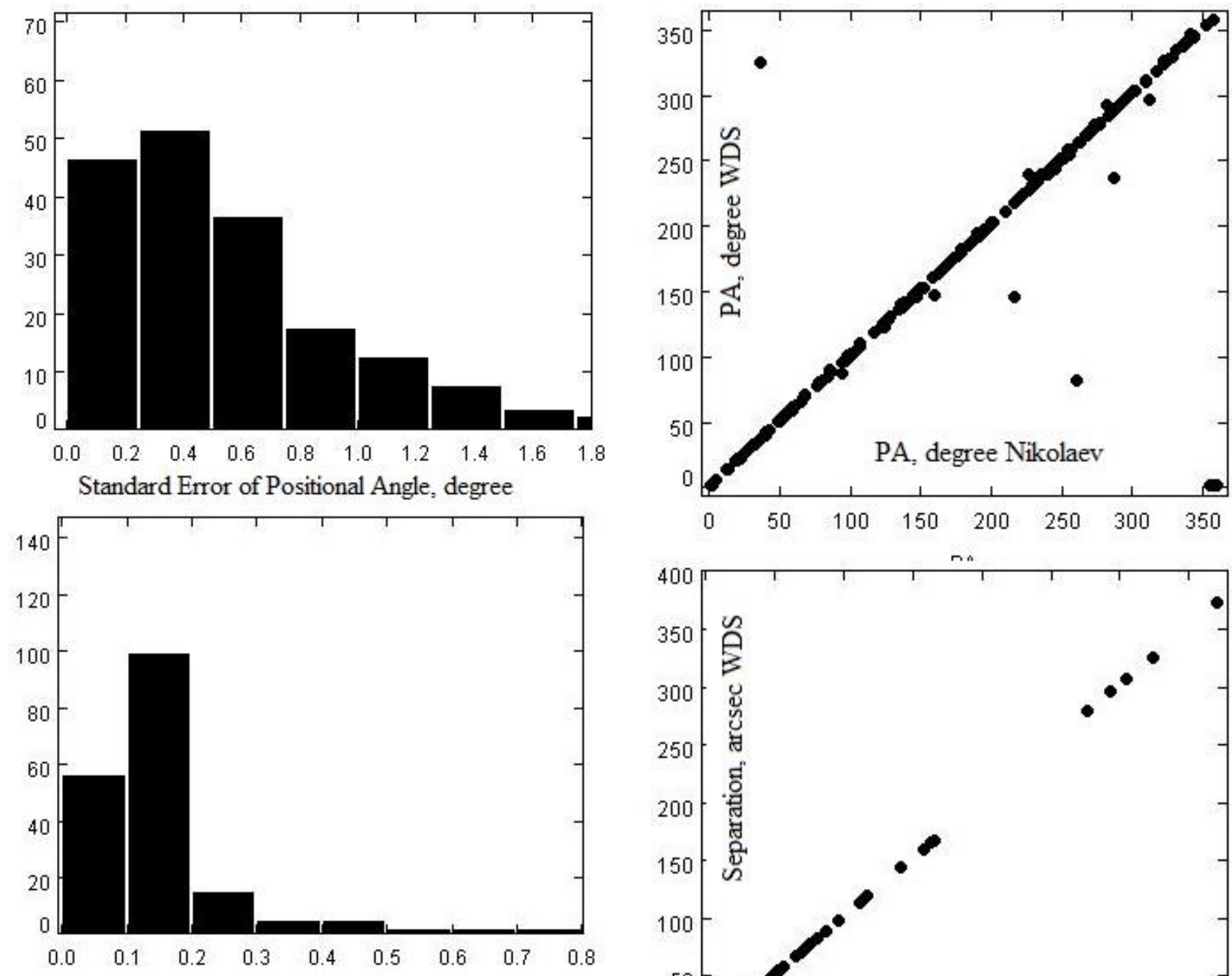

Standard Error of Separation, arcsec

Figure 3: Distribution of standard errors of measurements

We have compared our results with WDS data. It can be seen from figure 4 that they are in good accordance with each other.

\section{Conclusions}

The equatorial coordinates and proper motions of 361 WDS stars were obtained basing on CCD observations during 2013-2014 yr. Average accuracy of the cata$\log$ star position is about 30 mas in both coordinates. The new proper motions for WDS star were calculated with usage USNO A2.0 as the first epoch. Parameters for 199 WDS doubles (PA and separations) were obtained. The standard deviation is $0.5^{\circ}$ for PA and $0.15^{\prime \prime}$ for separation.

The results of measurements are going to be sent to WDS database.

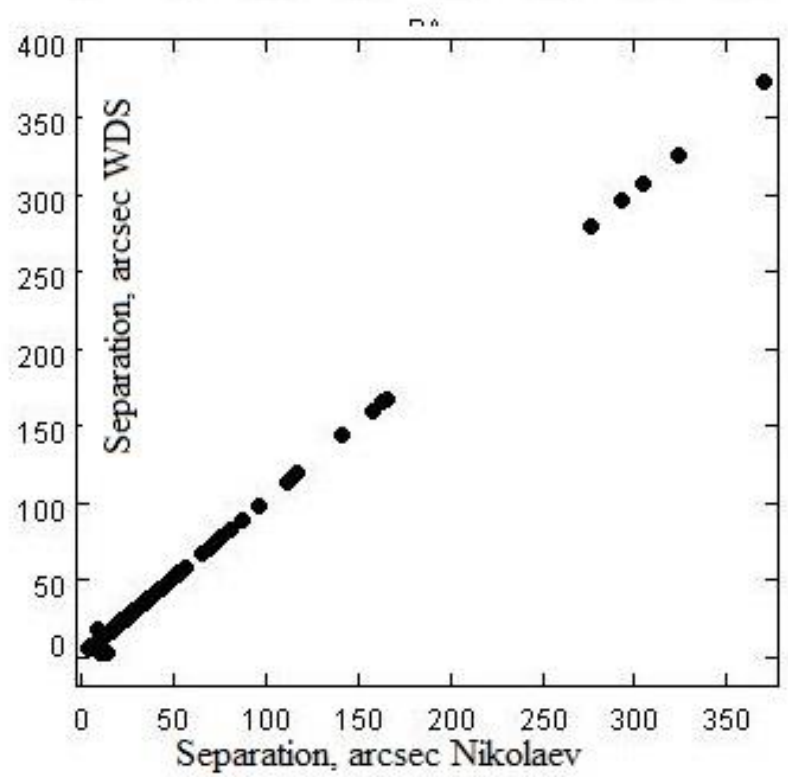

Figure 4: Measured values of separation and PA over separation and PA from WDS catalog (only double stars with separations between components more than about double FWHM were measured)

\section{References}

1. Mason B.D. et al: 2001, Astron. J., 122, 3466

2. http://www.astrometrica.at/

3. Zacharias N. et al: 2004, Astron. J., 127, 3043

4. Zacharias N. et al: 2013, Astron. J., 145, 44Z

5. http://www.star.bris.ac.uk/ mbt/topcat/

6. http://www.astrosurf.com/hfosaf/uk /tdownload.htm 